\title{
DYNAMICS OF THE RATIONAL DIFFERENCE EQUATION
}

$$
x_{n+1}=p x_{n}+\frac{q}{x_{n-1}^{2}}
$$

SK. SARIF HASSAN

This paper is dedicated to my PhD supervisor Prof. Pabitra Pal Choudhury, Former Professor of the Indian Statistical Institute, Kolkata, India.

Abstract. A second order rational difference equation

$$
x_{n+1}=p x_{n}+\frac{q}{x_{n-1}^{2}}
$$

with the parameters $p$ and $q$ which lies in $(0,1)$, is studied. The dynamics of the equilibrium is characterized through the trichotomy of the parameter $p<\frac{1}{2}, p=\frac{1}{2}$ and $p>\frac{1}{2}$. It is found that there is no periodic solution of period 2 and 3 but there exists periodic solutions with only periodic solution 5 and 10 are achieved computationally.

\section{INTRODUCTION}

The large number of open problems and conjectures in rational difference equations are still open to for research investigators. In the past three decades, the research is rational difference equations has been significantly proceeded. We therefore do not feel to include basic textbook introductory materials as they are easily available in the references $[1,2,3,4,5,6,7,8,9,10,11,12,13,14,15,16]$. We immediately proceed to the technicalities of the present rational difference equation which is of our interest.

\section{The Rational Difference Equation and Its Equilibrium}

Here the following second order rational difference equation (RDE) with the parameters $p$ and $q$ which lies in the interval $(0,1)$, is considered.

$$
x_{n+1}=p x_{n}+\frac{q}{x_{n-1}^{2}}
$$

We are primarily concerned with the boundedness nature of solutions, the stability of the equilibrium(s), the periodic and non-periodic character of the solutions of the $(2.1)$.

Date: April 6, 2020.

2000 Mathematics Subject Classification. Primary 39A10, 39A11, 39A20.

Key words and phrases. Rational Difference Equation, Asymptotic Stability, Periodic Solutions, Chaos and Fractal. 
2.1. Forbidden Set and Good Set. Assume that the parameters $p$ and $q$ are real numbers that belong to $(0,1)$. Determine the set $I$ of real initial values $x_{1}, x_{0}$ for which the difference equation (2.1) is well defined for all $n \geq 0$, and investigate the character of solutions of difference equation (2.1) with $x_{1}, x_{0} \in I$.

Let $\operatorname{Dom}(f)$ be domain of function $f\left(x_{n}, x_{n-1}\right)$ of the difference equation (2.1). Note that the function $f$ is required to be twice differentiable with continuous partial derivatives. If there exist initial values $\left(x_{0}, x_{1}\right)$ and such $n \in \mathbb{N}$ for which difference equation (2.1) is well defined and in iteration $n+1$ difference equation $(2.1)$ becomes equal to zero then initial values $\left(x_{0}, x_{1}\right)$ belong to forbidden set $\mathcal{F}$ and the complement of the forbidden set is named as Good set $[17,18]$. In the present study the function $f(u, v)=p u+\frac{q}{v^{2}}$ becomes equal to zero provided $v=-\sqrt{-\frac{q}{p u}}$ and $u<0$. Since we consider only non-negative initial values hence the forbidden set $\mathcal{F}$ is empty and therefore the good set is $(0, \infty)$.

The only real equilibrium point of the RDE Eq.(2.1) is $\frac{\sqrt[3]{q}}{\sqrt[3]{1-p}}$ which is non-negative $p, q \in(0,1)$.

Before we proceed further we present the most useful stability theorem $[1,5]$ as follows:

Theorem 2.1. (The Linearized Stability Theorem) Assume that the function F is a continuously differentiable function defined on some open neighbourhood of an equilibrium point $\bar{x}$ of the Then the following statements are true:

- When all the roots of the characteristic equation Eq.(3.2) have absolute value less than one, then the equilibrium point of Eq.(2.1) is locally asymptotically stable.

- If at least one root of the characteristic equation Eq.(3.2) has absolute value greater than one, then the equilibrium point of the RDE Eq.(2.1) is unstable.

- The equilibrium point of the RDE Eq.(2.1) is called hyperbolic if no root of Eq.(3.2) has absolute value equal to one. If there exists a root of the Eq.(3.2) with absolute value equal to one, then the equilibrium is called non-hyperbolic.

- An equilibrium point of the RDE Eq.(2.1) is called a saddle point if it is hyperbolic and if there exists a root of the Eq.(3.2) with absolute value less than one and another root of Eq.(1.2.2) with absolute value greater than one.

- An equilibrium point RDE Eq.(2.1) is called a repeller if all roots of the Eq.(3.2) have absolute value greater than one.

\section{Linearized Stability ANAlysis}

The linearized equation of the $\operatorname{RDE}(2.1)$ about the equilibrium point $\frac{\sqrt[3]{q}}{\sqrt[3]{1-p}}$ is

$$
y_{n+1}=p y_{n}-2(1-p) y_{n-1}
$$

The characteristic equation about the equilibrium point $\frac{\sqrt[3]{q}}{\sqrt[3]{1-p}}$ is

$$
\lambda^{2}-p \lambda+2(1-p)=0
$$


DYNAMICS OF THE RATIONAL DIFFERENCE EQUATION $x_{n+1}=p x_{n}+\frac{q}{x_{n-1}^{2}}$

The roots of the characteristic equation Eq.(3.2) are...

$$
\frac{1}{2}\left(p \pm \sqrt{p^{2}+8 p-8}\right)
$$

Remark 3.1. The roots of the characteristic equation Eq.(3.2) would be real if and only if

$$
p>2 \sqrt{6}-4
$$

Theorem 3.2. The equilibrium point $\frac{\sqrt[3]{q}}{\sqrt[3]{1-p}}$ where the parameter $q \in(0,1)$ is

- locally asymptotically stable if $\frac{1}{2}<p<1$

- non-hyperbolic if $p=\frac{1}{2}$

- hyperbolic provided $0<p<\frac{1}{2}$ or $\frac{1}{2}<p<1$

- a repeller if $0<p<\frac{1}{2}$

Proof. The proof follows from the Theorem 2.1 and it is left to the reader.

Remark 3.3. The equilibrium point $\frac{\sqrt[3]{q}}{\sqrt[3]{1-p}}$ is not unstable since none of the absolute value of the roots of the characteristic equation is greater than one.

Here a set of 5000 parameters $(p, q)$ are assembled in the two dimension so that the equilibrium point $\frac{\sqrt[3]{q}}{\sqrt[3]{1-p}}$ is locally asymptotically stable and a repeller respectively. The parameters are plotted in the following Fig.1.
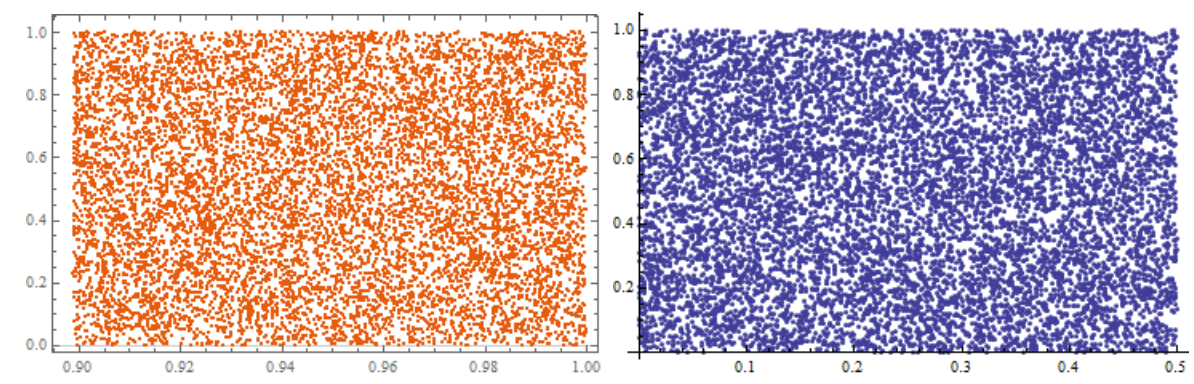

Figure 1. Parameters $(p, q)$ in the two dimension such that the $\frac{\sqrt[3]{q}}{\sqrt[3]{1-p}}$ is locally asymptotically stable (left) and a repeller (right).

Here we present few examples illustrating the dynamical behaviour of the system (2.1) as follows.

Example 3.4. Consider the parameters $p=0.549 \geq \frac{1}{2}, q=0.502$ the equilibrium is locally asymptotically stable and converges to 1.04 as shown in the Fig.2. 


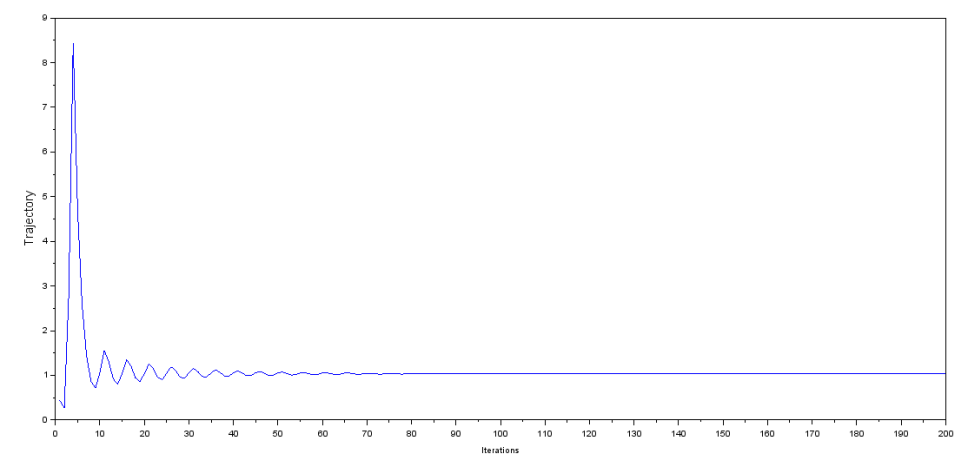

FiguRE 2. Locally asymptotically stable trajectory for the parameters $p=0.549$ and $q=0.502$.

Example 3.5. Consider the parameters $p=0.273 \leq \frac{1}{2}$ and $q=0.778$ the equilibrium 1.02 is unstable and makes a periodic trajectory with the periodic cycle 8.406 , $2.6198,0.7274,0.3123,1.5565,8.406$ with period 5 as shown in the Fig.3.

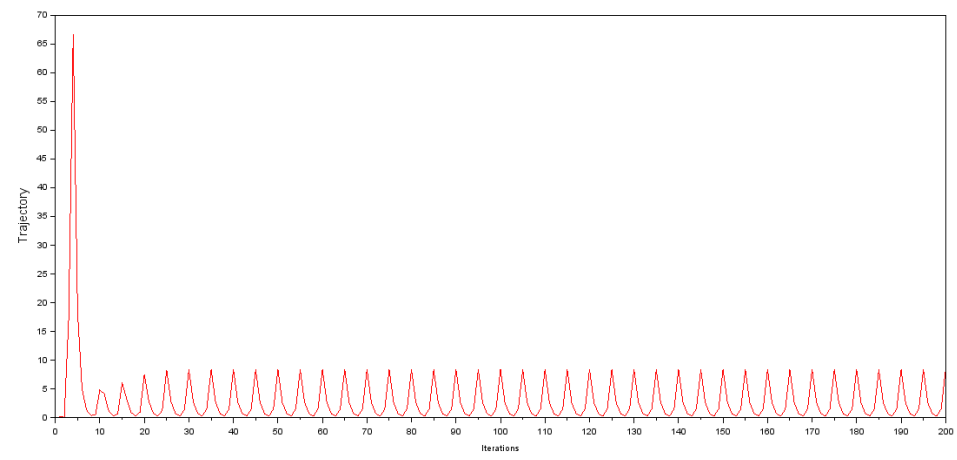

FiguRE 3 . Unstable equilibrium possesses to a periodic trajectory for the parameters $p=0273$ and $q=0.778$.

Example 3.6. Consider the parameters $p=0.5$ and $q=0.6325745$ the equilibrium 1.08 is a repeller and the trajectory gets away from the equilibrium as shown in the Fig.4. 
DYNAMICS OF THE RATIONAL DIFFERENCE EQUATION $x_{n+1}=p x_{n}+\frac{q}{x_{n-1}^{2}}$

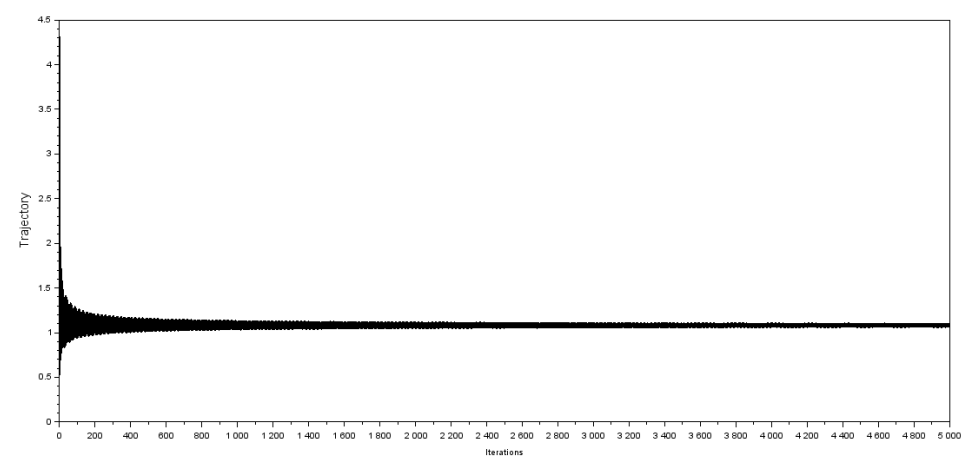

Figure 4. The equilibrium 1.08 is a repeller for the parameters $p=0.5$ and $q=0.6325745$.

3.1. Bounded and Convergence Results. Let us state a Lemma $[9,10,11]$ which would be used to derive convergence results of the (2.1).

Lemma 3.7. Let $I$ be a set of real numbers and let $F: I \times I \rightarrow I$ be a function $F(u, v)$, which decreases in $u$ and increases in $v$. Then for every solution $\left\{x_{n}\right\}_{n=-1}^{\infty}$ of the equation

$$
x_{n+1}=F\left(x_{n}, x_{n-1}\right), n=0,1,2, \ldots,
$$

the subsequences $\left\{x_{2 n}\right\}_{n=0}^{\infty}$ and $\left\{x_{2 n+1}\right\}_{n=-1}^{\infty}$ of even and odd terms of the solution do exactly one of the following:

- they are both monotonically increasing,

- they both are monotonically decreasing,

- Eventually, one of them is monotonically increasing and the other is monotonically decreasing.

Theorem 3.8. Every solution of the equation $x_{n+1}=p x_{n}+\frac{q}{x_{n-1}^{2}}$ possesses two subsequences of odd and even terms of the solution possess one of the following.

- they are both monotonically increasing,

- they both are monotonically decreasing,

- Eventually, one of them is monotonically increasing and the other is monotonically decreasing.

Proof. Setting up as the Lemma 3.7 Here $F(u, v)=\frac{q}{u^{2}}+p v$. Clearly $F$ is decreasing in $u$ and increasing in $v$ for any parameters $p, q \in(0,1)$. Hence by the Lemma 3.7 the theorem is concluded.

Lemma 3.9. Let $I$ be a set of real numbers and let $F: I \times I \rightarrow I$ be a function $F(u, v)$, which is non-increasing in $u$ for each $v$ in $I$ and non-decreasing in $v$ for each $u$ in I. If the difference equation (2.1) has no prime period two solutions in $I$, then the system 2.1 has a unique equilibrium and every solution converges to to the equilibrium $[9,10,11]$.

Theorem 3.10. The rational difference equation (2.1) has a unique equilibrium and every bounded solution converges to the equilibrium. 
Proof. Setting as the Lemma 3.9, we have the function $F(u, v)=\frac{q}{u^{2}}+p v$ which is non-increasing in $u$ for each $v$ in $I$ and non-decreasing in $v$ for each $u$ in a closed internal in $\mathbb{R}$. It is rest to prove that the $(2.1)$ does not possess any prime period two solutions. If possible let there is a prime period two solutions $\phi, \psi, \phi, \psi, \ldots$. Then it must satisfy $\phi=F(\phi, \psi)$ and $\psi=F(\psi, \phi)$. Solving these two system of equations for $\phi$ and $\psi$, the solutions boil down to no solutions in $\mathbb{R}$ other than the positive equilibrium $\frac{\sqrt[3]{q}}{\sqrt[3]{1-p}}$. Hence there is no prime period two solution of the (2.1). Therefore by the Lemma 3.9, the theorem is followed.

Theorem 3.11. Every positive solution of the (2.1) is bounded from above and below by positive constants.

Proof. Clearly, we can estimate equation (2.1) from below by

$$
x_{n+1}=p x_{n}+\frac{q}{x_{n-1}^{2}} \geq p c
$$

for some $c \in \mathbb{R}$.

Set that $p c \leq x_{n}$ and in equation (2.1) replacing it with a smaller value, the fraction increases and we get the estimation from above

$$
x_{n+1}=p x_{n}+\frac{q}{x_{n-1}^{2}} \leq p c+\frac{q}{p^{2} c^{2}}
$$

Finally we have

$$
p c \leq x_{n+1} \leq p c+\frac{q}{p^{2} c^{2}}
$$

for all $n \in \mathbb{N}$ and for some $c \in \mathbb{R}$. Hence the theorem is concluded.

\section{Periodic and Unstable Solutions}

In this section we shall study the unstable solutions of the rational difference equation (2.1). Study of such solutions are previously made in many articles which include $[19,20,21,22,23]$. In the previous section, it is already shown that difference equation (2.1) has no periodic solutions with period 2 and difference equation. In fact there is no period three solutions of the equation (2.1).

Theorem 4.1. There does not exist prime three period of the rational difference equation (2.1).

Proof. On contrary, assume that there is a prime period three solution $\phi, \psi, \xi, \phi, \psi, \xi, \ldots$ of the equation (2.1). Then the solution satisfy the following three equations:

$$
\begin{aligned}
\phi & =p \psi+\frac{q}{\xi} \\
\psi & =p \xi+\frac{q}{\phi} \\
\xi & =p \phi+\frac{q}{\psi}
\end{aligned}
$$

The above system of equations does not possess any solution in $\mathbb{R}$ other than the equilibrium of the equation (2.1). Hence the period three solution does not exist.

But the system (2.1) does possess high periodic solutions as shown through some examples below: 
DYNAMICS OF THE RATIONAL DIFFERENCE EQUATION $x_{n+1}=p x_{n}+\frac{q}{x_{n-1}^{2}} \quad 7$

Example 4.2. Consider the parameters $p=0.110517$ and $q=0.9329616$ then the equilibrium 1.02 is unstable and possesses a period $5\{8.441,0.933,0.116,1.084,69.120\}$. For any initial values near the equilibrium 1.02 is possessing towards the period 5 solution as shown in the trajectory is shown in the Fig.5.

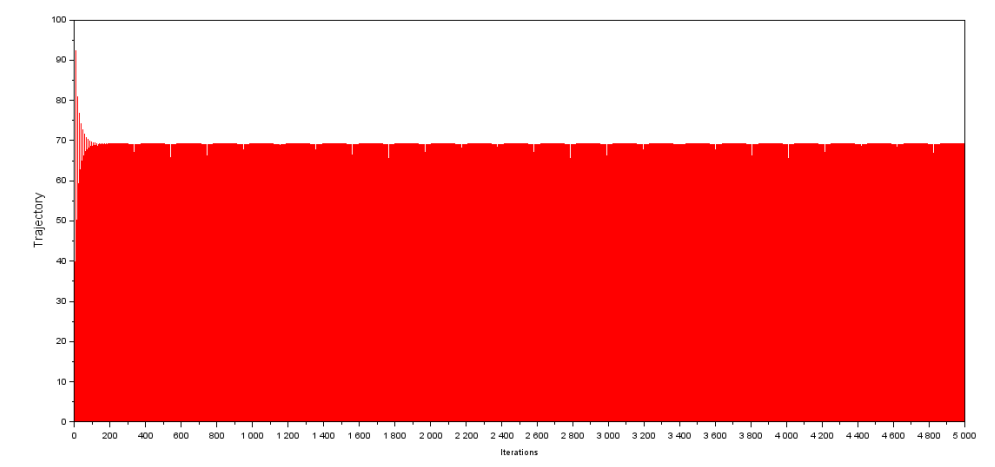

Figure 5. Period 5 trajectory for the parameters $p=0.110517$ and $q=0.9329616$.

Example 4.3. Consider the parameters $p=0.0487702$ and $q=0.4094825$ then the equilibrium 0.755 is unstable and possesses a period $10119.90045,10.555954$, $0.5148445,0.0287839,1.5462431,494.31254,24.278991,1.184093,0.0584431,0.2949047$. For any initial values near the equilibrium 1.02 is possessing towards the period 10 solution as shown in the trajectory is shown in the Fig.6.

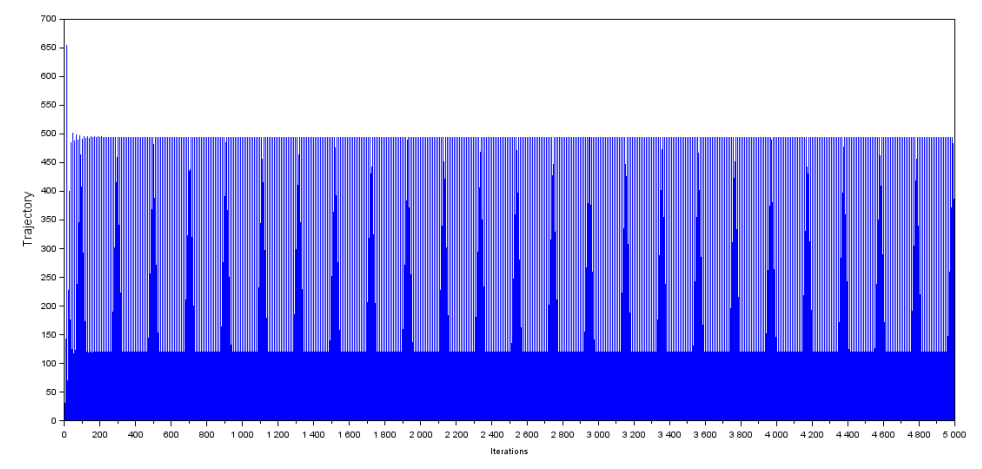

FiguRE 6. Period 10 trajectory for the parameters $p=0.0487702$ and $q=0.4094825$.

Example 4.4. Consider the parameters $p=0.4566879$ and $q=0.8833888$ then the equilibrium 1.18 is unstable and possesses unstable non-periodic solution. For any 
initial values near the equilibrium 1.18 is possessing unstable non-periodic solutions as shown in the trajectory with 200000 iterations is shown in the Fig.7.

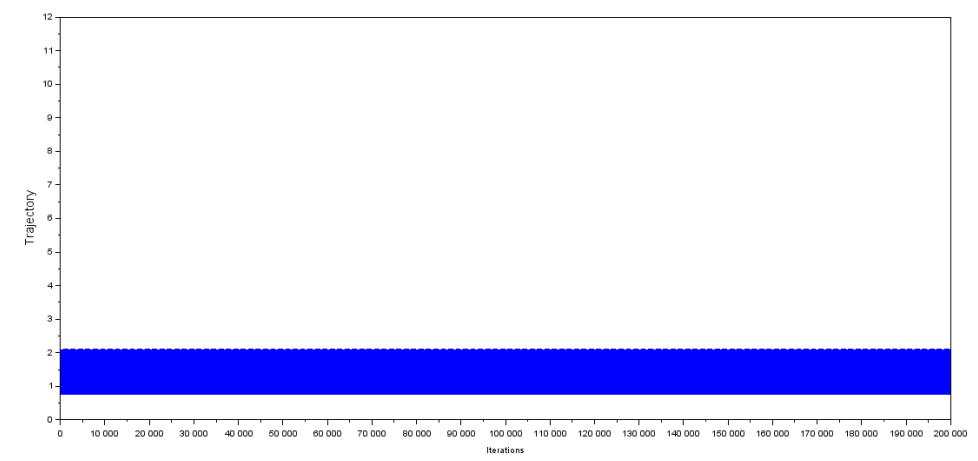

FiguRE 7. Unstable non-periodic trajectory for the parameters $p=0.4566879$ and $q=0.8833888$.

Example 4.5. Consider the parameters $p=0.3990529$ and $q=0.4829179$ then the equilibrium 0.93 is unstable and possesses unstable non-periodic solution. For any initial values near the equilibrium 0.93 is possessing unstable non-periodic solutions as shown in the trajectory with 200000 iterations is shown in the Fig.8.

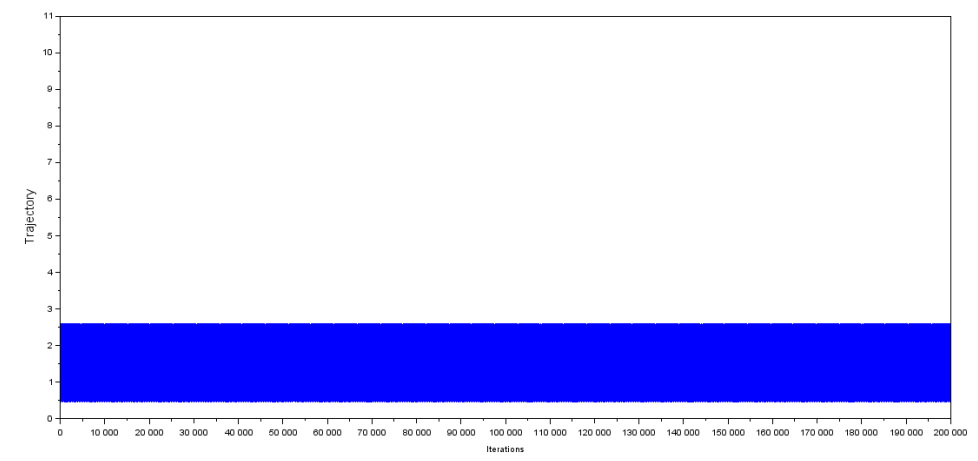

FiguRe 8. Unstable non-periodic trajectory for the parameters $p=0.3990529$ and $q=0.4829179$.

Example 4.6. Consider the parameters $p=0.4001402$ and $q=0.1205996$ then the equilibrium 0.586 is unstable and possesses unstable non-periodic solution (chaotic). For any initial values near the equilibrium 0.586 is possessing unstable non-periodic solutions as shown in the trajectory with 200000 iterations is shown in the Fig.9. 
DYNAMICS OF THE RATIONAL DIFFERENCE EQUATION $x_{n+1}=p x_{n}+\frac{q}{x_{n-1}^{2}}$

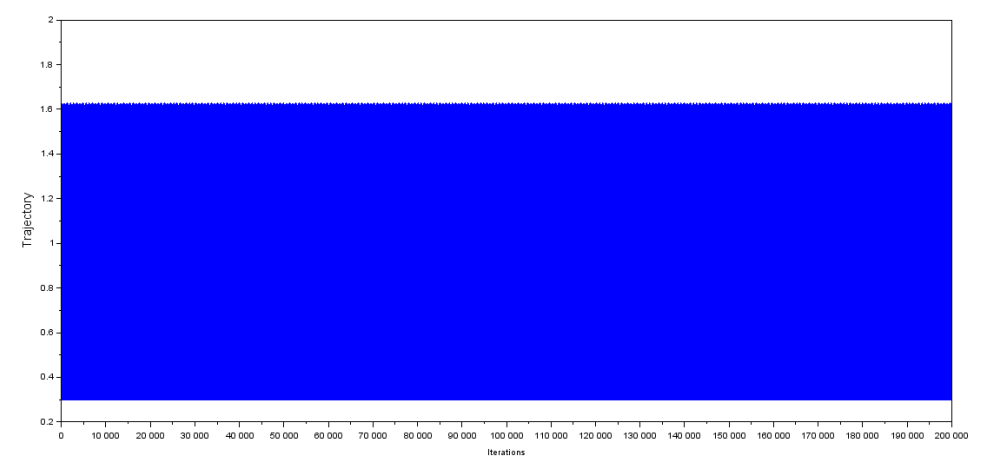

FIGURE 9. Unstable non-periodic trajectory for the parameters $p=0.4001402$ and $q=0.1205996$.

Example 4.7. Consider the parameters $p=0.5$ and $q=0.0437334$ then the equilibrium 0.444 is non-hyperbolic and its corresponding trajectory with 20000 iterations is shown in the Fig. 10.

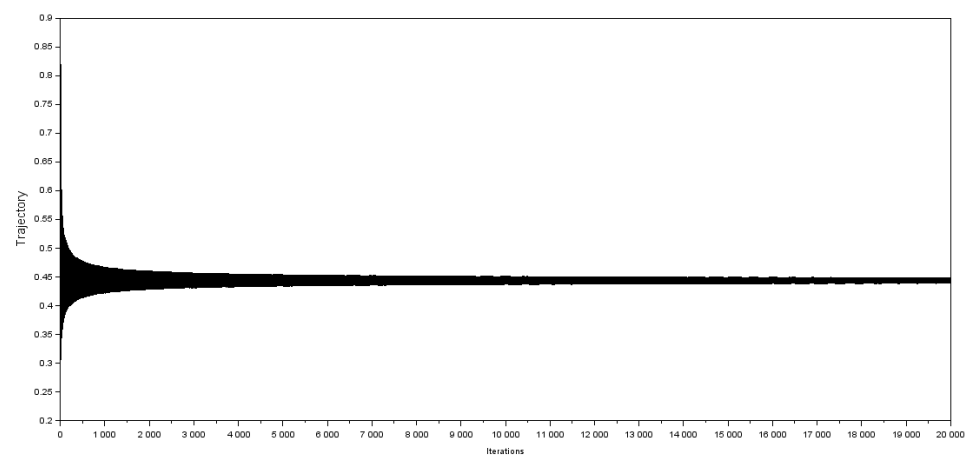

FiguRE 10. Non-hyperbolic trajectory for the parameters $p=0.5$ and $q=0.0437334$.

Example 4.8. Consider the parameters $p=0.5$ and $q=0.4498763$ then the equilibrium 0.965 is non-hyperbolic and its corresponding trajectory with 20000 iterations is shown in the Fig.11. 


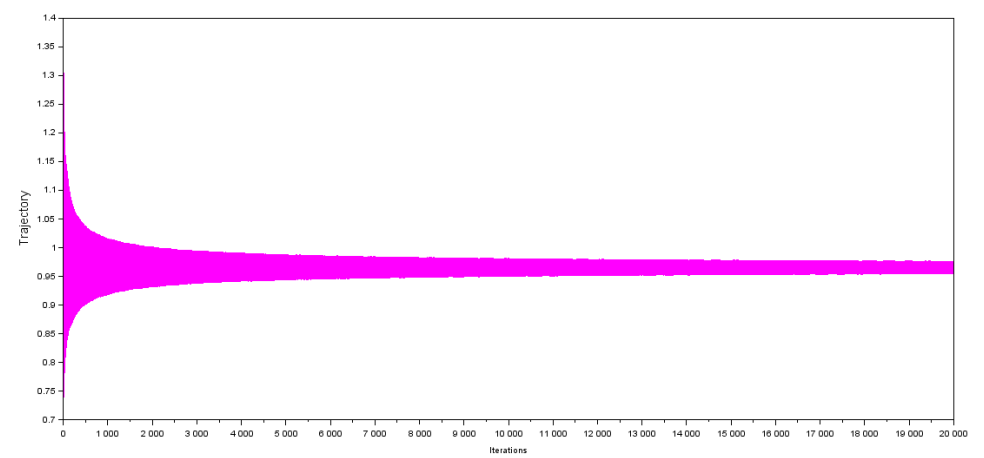

FiguRE 11. Non-hyperbolic trajectory for the parameters $p=0.5$ and $q=0.4498763$.

Remark 4.9. Other than the periodic solution of the RDE (2.1) with periods 5 and 10 , no periodic solution are achieved even after an extensive run of the code for finding periodic solutions.

Remark 4.10. The computational observation suggests that the periodic solutions depicted are stable periodic solutions.

\section{REFERENCES}

1. Kulenovic, M. R., \& Ladas, G. (2001). Dynamics of second order rational difference equations: with open problems and conjectures. Chapman and Hall/CRC.

2. Touafek, N. (2012). On a second order rational difference equation. Hacettepe Journal of Mathematics and Statistics, 41(6), 867-874.

3. Hu, L. X., Li, W. T., \& Stević, S. (2008). Global asymptotic stability of a second order rational difference equation. Journal of Difference Equations and Applications, 14(8), 779-797.

4. Elsayed, E. M. (2012). Solutions of rational difference systems of order two. Mathematical and Computer Modelling, 55(3-4), 378-384.

5. Elsayed, E. M., El-Dessoky, M. M., \& Asiri, A. (2014). Dynamics and behavior of a second order rational difference equation. J. Comput. Anal. Appl, 16(4), 794-807.

6. Chatterjee, E., \& Hassan, S. S. (2018). On the asymptotic character of a generalized rational difference equation. Discrete and Continous Dynamical Systems, 38(4), 1707-1718.

7. Kalabušić, S., \& Kulenović, M. R. S. (2004). Rate of convergence of solutions of rational difference equation of second order. Advances in Difference Equations, 2004(2), 901703.

8. Saleh, M., \& Abu-Baha, S. (2006). Dynamics of a higher order rational difference equation. Applied Mathematics and Computation, 181(1), 84-102.

9. Amleh, A. M., Camouzis, E., \& Ladas, G. (2007). On second-order rational difference equations, part 1. Journal of Difference Equations and Applications, 13(11), 969-1004.

10. Huang, Y. S., \& Knopf, P. M. (2004). Boundedness of positive solutions of second-order rational difference equations. Journal of Difference Equations and Applications, 10(11), 935940.

11. Li, X. (2005). Qualitative properties for a fourth-order rational difference equation. Journal of Mathematical Analysis and Applications, 311(1), 103-111.

12. Stević, S., Diblík, J., Iričanin, B., \& Šmarda, Z. (2014). On a solvable system of rational difference equations. Journal of Difference Equations and Applications, 20(5-6), 811-825.

13. Abo-Zeid, R. (2010). Global asymptotic stability of a second order rational difference equation. Journal of applied mathematics \& informatics, 28(3-4), 797-804. 
DYNAMICS OF THE RATIONAL DIFFERENCE EQUATION $x_{n+1}=p x_{n}+\frac{q}{x_{n-1}^{2}}$

14. Patula, W., \& Voulov, H. (2003). On the oscillation and periodic character of a third order rational difference equation. Proceedings of the American Mathematical Society, 131(3), 905909.

15. Taskara, N., Uslu, K., \& Tollu, D. T. (2011). The periodicity and solutions of the rational difference equation with periodic coefficients. Computers \& Mathematics with Applications, 62(4), 1807-1813.

16. Dannan, F., Elaydi, S., \& Liu, P. (2000). Periodic solutions of difference equations. Journal of difference equations and applications, 6(2), 203-232.

17. Yazlik, Y., Tollu, D. T., \& Taskara, N. (2013). On the solutions of difference equation systems with Padovan numbers. Applied Mathematics, 4(12), 15.

18. Stevic, S. (2004). More on a rational recurrence relation. Appl. Math. E-Notes, 4(1), 80-85.

19. Stević, S. (2004). Periodic character of a class of difference equation. Journal of Difference Equations and Applications, 10(6), 615-619.

20. Stević, S. (2006). A note on periodic character of a higher order difference equation. In Rostocker Mathematisches Kolloquium (No. 61, pp. 21-30).

21. Elsayed, E. M. (2011). Solution and attractivity for a rational recursive sequence. Discrete Dynamics in Nature and Society, 2011.

22. Li, W. T., \& Sun, H. R. (2005). Dynamics of a rational difference equation. Applied Mathematics and Computation, 163(2), 577-591.

23. Berg, L. (2002). On the asymptotics of nonlinear difference equations. Zeitschrift für Analysis und ihre Anwendungen, 21(4), 1061-1074.

Department of Mathematics, Pingla Thana Mahavidyalaya, Maligram, Paschim MeDinipur, 721140, WeSt Bengal, India

Email address: sarimif@gmail.com 